Review

\title{
Microgravity, Bone Homeostasis, and Insulin-like Growth Factor-1
}

J. Kelly Smith

Departments of Academic Affairs and Biomedical Sciences, James H. Quillen College of Medicine, East Tennessee State University, PO Box 70300, Johnson City, TN 37614

Correspondence: smithj@etsu.edu; Tel: 4237947425 
Abstract: Astronauts at are risk of losing 1.0 - 1.5\% of their bone mass for every month they spend in space despite their adherence to high impact exercise training programs designed to preserve the musculoskeletal system. This article reviews the basics of bone formation and resorption and details how exposure to microgravity or simulated microgravity affects the structure and function of osteoblasts, osteocytes, osteoclasts, and their mesenchymal and hematologic stem cell precursors. It details the critical roles that insulin-like growth facor-1 and its receptor IGFR1 play in maintaining bone homeostasis and how exposure of bone cells to microgravity affects the function of these growth factors. Lastly, it discusses the potential of tumor necrosis factor-related apoptosis-inducing ligand, syncytin-A, and sclerostin inhibitors and recombinant IGF-1 as a bone-saving treatment for astronauts in space and during their colonization of the Moon.

Keywords: Insulin-like growth factor-1; Insulin-like growth factor-1 receptor; microgravity; osteoblasts; osteocytes; osteoclasts; IGF-1; IGF1R; rIGF-1 


\section{Introduction}

All terrestrial forms of life have had millions of years to adapt to Earth's gravity $\left(9.8 / \mathrm{m} / \mathrm{sec}^{2}\right)$. Cytoskeletons constructed with actin, microfilaments and microtubules resist compressive forces and allow internal load bearing. Adhesive molecules such as integrins deal with external compressive forces and allow cells to maintain their positions in space. Mechanosensors shuttling between cytoplasmic membranes and nuclei and matrix clusters of cell extension kinases keep the cell informed of needed changes in gene expression. Gravisensing organelles inform roots and stems which direction in space they should occupy. And cells use thermal convection in which heated fluids rise to the top of the gravity vector and are then exchanged by cooler fluids, establishing a convection current that dissipates heat, renews nutrient supplies, and removes waste materials.

Nature is the ultimate architect and has done well by its creatures on Earth. But man has thrown nature a curve ball and ventured into space where all these adaptations are obsolete.

The adverse effects of microgravity are particularly severe on the musculoskeletal system, particularly bone. In this review, I detail the effects of microgravity on bone homeostasis and review the prominent roles that insulin-like growth factor- 1 and its receptor, IGF-1R, play in the growth and maintenance this vital tissue.

\section{Bone Formation}

In its initial phase mesenchymal stem cells and osteoblasts occupying the surface of cortical bone, secrete receptor activator of nuclear factor kappa B ligand (RANKL) which upregulates osteoclast differentiation by binding to RANK receptors on the surface of osteoclast precursors. This process is counterbalanced by osteoprotegerin (OPG), a soluble decoy receptor that inhibits osteoclastogenesis by binding RANKL. In the second phase, newly evolved osteoclasts secrete hydrochloric acid and acid phosphatases that degrade surface bone and the bone matrix, releasing imbedded growth factors, including bone morphogenic proteins, transforming growth factor- $\beta$ and insulin-like growth factor. Osteoclasts form Howship's lacunae in trabecular bone and a cutting zone in cortical bone; once these cavities reach a certain size, osteoclasts undergo apoptosis, terminating bone resorption. In the third phase of bone remodeling, the newly liberated growth factors stimulate osteoblasts to evolve from their mesenchymal stem cell precursors to control bone mineralization and secrete collagen to form the bone matrix. In the fourth phase of bone remodeling, osteoblasts trapped in the bone matrix evolve into osteocytes, cells that connect with one another, osteoblasts, and osteoclasts through a myriad of dendritic processes that constitute the lacunar-canalicular network (see figure 1). Osteocytes are the most abundant cell type in bone (90-95\% of all bone cells); they respond to hormonal and mechanical signals and are the primarily cell responsible for the control of bone homeostasis [1,2]. 
Figure 1.

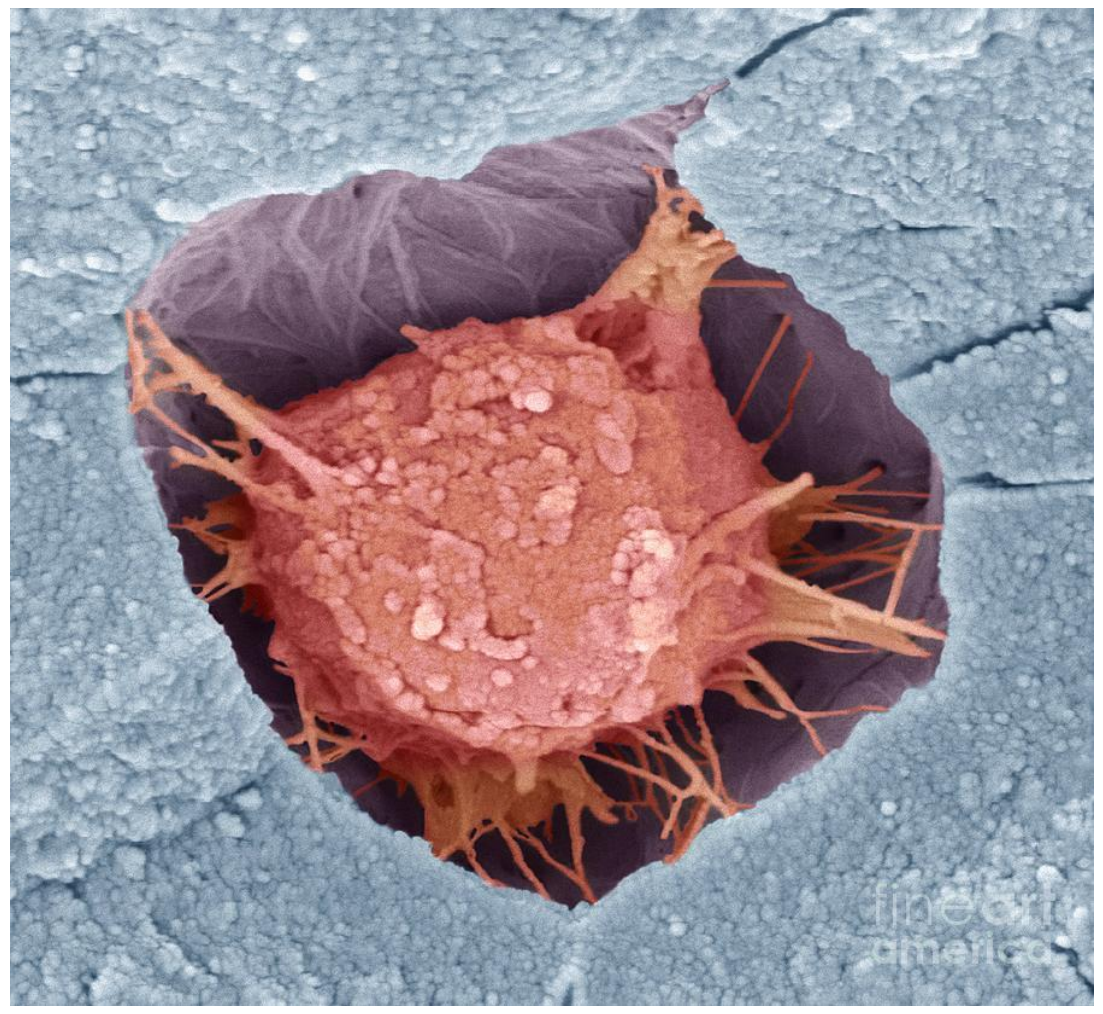

A photomicrograph of an osteocyte embedded in its lacuna. Evolved from osteoblasts entrapped in bone matrix, osteocytes form a multitude of dendritic extensions that communicate with osteoblasts, osteoclasts, and other osteocytes through a network of channels cut into the bone matrix. These dendritic extensions are sensitive to pressure changes associated with mechanical loading and unloading and allow osteocytes to regulate the balance between bone absorption and bone formation. Osteocytes undergo apoptosis in zero gravity, losing their control over bone homeostasis, and secreting sclerostin, a mechanosensitive protein that inhibits bone formation and enhances bone resorption (photograph by Steve Gschmeissner).

\section{Mechanotransduction}

\subsection{Osteocytes}

Exposure of osteocytes to shear stress generated in blood vessels within the Volkmann and Haversian canals and interstitial fluid in the lacunar-canalicular network and in spaces between the crystallites of the mineral hydroxyapatite and collagen fibers distorts their shape and activates the Wnt signaling pathway via translocation of $\beta$-catenin to the nucleus [2]. Wnt signaling is critical for bone mass accrual, bone remodeling, and fracture repair, and loss of function in Wnt or in mutations of its co-receptor LRP5 result in low bone mass and an increase in fragility fractures [2-4]. Cell shape distortion involves cytoskeletal rearrangement of actin fibers and microtubules, both of which are known to be integral players in mechanotransduction. Osteocyte cilia, dendritic extensions, and integrins in the extracellular matrix also act as mechanosensors [4, 5]. Hillsley and Frangos propose that the bone loss observed in disuse and microgravity is due to a lack or decreased interstitial fluid flow [6] 
Liu and associates have shown that osteocytes also respond to changes in hydraulic pressure. Cultures of osteocyte-like MLO-Y4 cells sensed cyclic changes in hydraulic pressure and responded by increasing intracellular calcium concentrations, COX-2 levels, and RANKL/osteoprotegerin mRNA ratios, and by altering cytoskeleton organization of actin and microtubules [7]. Mobilization of intracellular calcium activates downstream signaling of protein kinase A (PKA), MAPK, c-Fos, and prompts nuclear translocation of NF-KB. Blocking calcium release attenuates the mechanically induced upregulation of osteogenic gene expression in vitro and abrogates load-induced bone formation in vivo. COX-2 is the gene that encodes cyclooxygenase-2, a mediator of prostaglandins that play a role in bone homeostasis $[7,8]$.

\subsection{Osteoblasts}

Young and associates found that reduced levels of osteoblast mechanostressors activates mechanotransduction via focal adhesion kinase (FAK) contained within extracellular matrix signaling complexes. Osteoblasts respond by increasing the expression of proteins involved in bone formation, such as COX-2, c-Fos and osteopontin E2 [9]. Ban and associates found that osteoblasts subjected to low levels of fluid shear stress proliferated and increased their production of bone markers including alkaline phosphatase and osteocalcin [10].

\subsection{Osteoclasts}

Osteoclast responses to mechanical loading are mediated by osteoblasts and osteocytes. Mechanically stimulated osteoblasts inhibit osteoclastogenesis by producing protegerin; this decoy receptor binds RANKL and prevents it from ligating receptors on osteoclast hematologic stem cells. Osteocytes control osteoclast activity by secreting sclerostin, a mechanosensing protein that increases bone resorption while decreasing bone formation [11].

Other structures within bone may also play a role in the response of bone to mechanical forces, including ion channels, connexins, integrins, and the glycocalyx $[2,5]$.

\section{Mechanotransduction in Space $\left(1 \times 10^{-6} \mathrm{~g}\right)$}

\subsection{Osteocytes}

Osteocytes are particularly susceptible to the adverse effects of zero gravity. In mice, one month of spaceflight decreased femoral trabecular bone volume by $64 \%$ and increased bone resorption by $140 \%$ when compared to ground controls. These changes were associated with osteocyte apoptosis, reductions in osteocyte lacunar volumes, and increases in lacunar vacancies (+ 344\% vs ground controls); fatty metamorphosis of mesenchymal stem cell precursors was also observed [12]. Similar findings were noted in iliac crest biopsies of monkeys flown for 14 days at zero gravity [13].

\subsection{Osteoblasts}

A site-specific bone loss of 1-2\% occurs in astronauts and in-flight animals after 1 month of spaceflight. The loss is associated with changes in osteoblast cellular and nuclear morphology and altered mRNA expression of type I collagen, osteocalcin, and insulin-like growth binding proteins [14]. Nabavi and associates found that osteoblasts subjected to 5 days of microgravity had extended shapes, fragmented or condensed nuclei, shorter microtubules, and smaller and fewer focal adhesions [15]. 
Hughs-Fulford found that serum-activated osteoblasts had a $60 \%$ reduction in growth as compared to ground controls, presumably due to alterations in growth factor receptors and/or cell-connected matrix kinase pathways. After 4 days in space there was a loss of focal adhesions and a complete collapse of the osteoblast cytoskeleton. He postulated that the collapse was due to F-actin microfilament failure and/or inhibition of microtubular polymerization [16]. The same investigator had previously reported that cultured osteoblasts had increased levels of C-fos mRNA and diminished levels of Cox-2 mRNA [17]. C-fos is a protooncogene that converts extracellular signals into changes in gene expression; Cox-2 catalyzes the rate-limiting step in prostaglandin synthesis. Guignandon and associates also reported that osteoblastic integrin-mediated cell adhesion was disrupted in cells flown for six days on the space shuttle [18]

\subsection{Osteoclasts}

Differentiated osteoclasts cultured on bone substrates for 10 days of zero gravity increased their expression of genes involved in osteoclast maturation and bone resorption when compared to ground controls [19].

\section{Mechanotransduction in Simulated Microgravity $\left(g \geq 10^{-3}\right)$}

\subsection{Osteocytes}

Robling and associated investigated the mechanoregulation of sclerostin and its regulatory gene Sost in rodent osteocytes under enhanced (ulnar loading) and reduced (hindlimb unloading) conditions. Sost transcripts and sclerostin protein levels were reduced in loaded bone and increased in unloaded bone [11]. Lin and associates found that mechanical unloading of wildtype mice caused a decrease in Wnt/beta-catenin signaling accompanied by increases in Sost and sclerostin production and reductions in bone formation and in the viability of osteocytes and osteoblasts [20].

\subsection{Osteoblasts}

Using NASA's Rotary Cell Culture System, Saxena and associates found that modeled microgravity inhibited osteoblastogenesis and increased adipose differentiation in human stem cells. The transformation involved reductions in RhoA activity and cofilin phosphorylation, disruption of F-actin stress fibers, and decreased integrin signaling through focal adhesion kinase [21]. Employing the same culture system, Ontiveros and McCabe reported a 50-80\% reduction in alkaline phosphatase, Runx2, osteocalcin expression, and AP-1 transactivation in cultures of rat osteoblasts [22], and Patel and associates noted that 3 days of rotation altered the expression of 61 mechanosensitive genes, including osteomodulin, Runx2 and osteoglycin in cultures of mouse tibial preosteoblasts [23].

Using two-axis random positioning machines, Shi and associates found that simulated microgravity inhibited rat calvarial osteoblast differentiation, maturation, and ciliogenesis and attributed the change to atrophy of osteoblast cilia [24]. Sun and associates found that simulated microgravity reduced intracellular free calcium levels by inhibiting calcium channels in mouse osteoblasts [25]. As previously noted, low levels of intracellular calcium impairs osteogenic gene expression in vitro and abrogates load-induced bone formation in vivo. Dai and associates found that simulated microgravity inhibited the proliferation and osteogenesis of rat bone marrow mesenchymal stem cells [26]. Using cultures of human bone marrow mesenchymal stem cells, Li and associates found that simulated microgravity 
inhibited genes regulating proliferation and differentiation while upregulating genes involved in adipogenesis [27]. Chen and associates found that simulated microgravity inhibited osteogenic differentiation of mesenchymal stem cells by inhibiting the expression of transcriptional coactivator with PDZ-binding motif, an important regulator of osteogenesis [28]

Experiments measuring the metabolomics and proteomics of cultured human osteoblasts found that simulated microgravity caused a decrease in mitochondrial proteins, a reduction in mitochondrial energy potential, and an oxidative stress response (decreases in oxidized glutathione and antioxidant enzymes) [29].

\subsection{Osteoclasts}

Using NASA's rotary cell culture system and mouse bone marrow cultures, Sambandam and associates found that simulated microgravity enhanced the maturation of osteoclast precursors [30,31]. Ethiraj and associates reported similar findings noting that osteoclastogenesis was associated with increased stem cell expression of syncytin-A [32]. Rucci and associates found that modeled microgravity stimulated osteoclastogenesis and bone resorption by increasing osteoblast RANKL/osteoprotegerin ratios [33], and Saxena and associates found that modeled microgravity and hindlimb unloading sensitized osteoclast precursors to RANKL-mediated osteoclastogenesis [34].

\section{The Somatotrophic Axis}

The somatotropic axis consists of hormonal circuits that regulate somatic growth, including body and bone mass, body adiposity, and lipid and carbohydrate metabolism. The axis includes growth hormonereleasing hormone (GHRH), growth hormone (GH), insulin-like growth factors (IGFs) 1 \& 2 and their receptor, IGF1R, six IGF binding proteins (IGFBPs), the acid-labile subunit (ALS), somatostatin, and ghrelin. Also included are growth hormone receptor's downstream mediators, Janus kinase 2 (JAK2), signal transducer and activator of transcription 5 (STAT5) and suppressors of cytokine signaling 1-3 (SOCS1-3) [1]. IGF-2 is primarily involved in the grow and development of preadolescents and will not be discussed in this article.

\subsection{Insulin-like growth factor-1 (IGF-1)}

IGF-1 is structurally related to insulin. It consists of 70 amino acids in a single chain with three intramolecular bridges and has a molecular weight of 7,649 Daltons [35, 36]. This growth factor is produced by GH-stimulated hepatocytes (the main source of circulating IGF-1) and by all nonhepatic tissues where it acts in a paracrine/autocrine fashion [1].

In serum, $75 \%$ of IGF-1 exists in a 150-kDa complex consisting of IGF, IGFBP-3 or -5 and the ALS (37). About $20 \%$ of IGF-1 is bound in binary complexes with IGFBPs, and $~ 5 \%$ is found in the free, biologically active, state, which has a half-life of $\sim 10$ minutes. IGFBP binding prolongs the half-life of IGF-1 and assists in its delivery to specific tissues [1].

When in close proximity to its receptor IGF-1 is liberated from its BP by the actions of one of the serine proteases, pregnancy-associated plasma protein-A or prostate-specific antigen. In tissue, kallikreins, cathepsins, and matrix metalloproteinases have been shown to assist in tissue remodeling and repair by cleaving the IGFBPs and releasing IGF-1 [1] 


\subsection{Insulin-like growth factor-1 receptor (IGF-1R)}

Although IGF-1 can bind to insulin receptors, its primary action is mediated when it binds to its main receptor, IGF-1R. IGF-1R is a class II receptor tyrosine kinase (RTK) which plays a critical role in cell growth and differentiation. Binding of one IGF-1 molecule is sufficient to break the autoinhibited state of IGF-1R and initiate receptor activation [27]. This results in the activation of a variety of intracellular transduction cascades including phosphatidlyinositol-3 kinase (PI3K) and its downstream target, mammalian target of rapamycin (mTOR). Recently, IGF-IR has been shown to have extensive cross-talk with many other receptors and can function as an RTK/GPCR hybrid. In addition, after activation, IGF-IR may translocate to the nucleus and function as a transcriptional cofactor; alternatively, it is recycled to the surface or degraded (38).

\section{IGF-1 and Bone Homeostasis}

IGF-1 is the most abundant growth factor in bone matrix [33] where it is synthesized by osteoblast mesenchymal precursors, mature osteoblasts, osteoclasts and osteocytes [1,35, 36]. Osteoblasts also produce all the IGFBPs [39]. Hepatocyte synthesized IGF-1 is distributed to the matrix via the bone's nutrient and periosteal arteries located within the Volkmann and Haversian canals.

In bone, IGF-1 regulates periosteal bone expansion (radial bone growth) by its effects on osteoprogenitors at the periosteal or endocortical bone surfaces. Linear bone growth is controlled by growth hormone which stimulates chondrocyte precursors to produce IGF-1 which then acts in an autocrine fashion to induce the production of type II collagen1a via activation of the phosphatidylinositol 3-kinase/phosphoinositide-dependent kinase-1 pathway [40]. With progression of chondrocytes to terminal differentiation, IGF1R signaling is terminated [37]. IGF-1 regulates bone mass by activation of mTOR in mesenchymal stem cell progenitors of osteoblasts [41].

IGF-1 has pro-differentiating and pro-survival effects on osteoblasts and their mesenchymal precursors and is essential for the anabolic actions of PTH on bone [42]. Activation osteoblast IGF1R initiates the expression of the runt-related transcription factor 2 (Runx2) which stimulates the transition of mesenchymal stem cells into osteoprogenitors. Downstream of Runx 2 is osterix (osx) which controls the maturation of osteoprogenitors into mature osteoblasts. Osteoblasts are responsible for the production and secretion of the extracellular matrix and expresses genes involved in matrix calcification [39].

Osteoclasts differentiate from mononuclear progenitors in the presence of macrophage colony stimulating factor (M-CSF) and RANKL. Wang and associates found that mRNA expression of RANKL, RANK, and M-CSF was diminished by $35-60 \%$ in IGF-1 knockout mice and led to a reduction in osteoclastogenesis; the results confirm the importance of IGF-1 in promoting osteoclast differentiation from hematologic stem cell precursors [43].

Both animal and human studies have shown that circulating levels and locally produced autocrine/paracrine sources of IGF-1 each play an important role in achieving peak bone mass and strength $[1,37]$.

\section{IGF-1 and Mechanical Loading}

In vitro experiments of cultured MC3T3-E1 osteoblast-line cells have shown that mechanical loading generated by interstitial fluid shear stress protects osteoblasts from tumor necrosis factor- $\alpha$-mediated 8 
apoptosis and that the protection is mediated by flow-enhanced IGF-1-rceptor signaling [44,45]. Osteoblast deletion of IGF-1 or IGF1R abates the osteogenic response to mechanical loading whereas transgenic overexpression of IGF-1 in osteoblasts results in an enhanced responsiveness to in vivo mechanical loading in mice. [46]

\section{IGF-1 and Mechanical Unloading}

Studies during spaceflight and in bone unloading experiments on Earth have shown that bone unloading suppresses IGF-1 production. Kumei et al found that spaceflight decreased mRNA levels of IGF-1 in cultures of rat osteoblasts as compared to ground controls; they also found that microgravity completely suppressed mRNA expression of the insulin receptor substrate-1, a postreceptor signaling molecule of IGF-1 [47]. Sakata and associates found that skeletal unloading induces resistance to IGF-1 by inhibiting the activation of IGF-signaling pathways including the expression of alphaVbeta3 integrin [48]. And Triplett et al found that mechanical loading by fluid shear stress enhanced IGF-1R signaling in osteoblasts whereas mechanical unloading inhibited its signaling [45]. Kostenuik and associates found that skeletal unloading caused resistance of osteoprogenitor cells to parathyroid hormone due to the development of resistance to IGF-1 [42].

Hind limb suspension in rats has been shown to inhibit IGF1R signaling pathways with consequent reduction in the number and differentiation of osteoblast progenitors and reduced bone formation; responsiveness to IGF1R could be restored with reloading $[21,34,46]$. In contrast, unloading of IGF-1R knockout mice caused a permanent loss of bone synthesis, indicating that IGF1 signaling is necessary for bone formation following disuse or zero gravity $[1,37]$.

\section{Discussion}

Many of the studies on bone homeostasis in space and in terrestrial models of microgravity are motivated by the need to find treatments capable of abrogating the striking degree of bone loss experienced by occupants of the space shuttle. Although most recommendations are non-specific, a few have suggested specific targets to investigate. These include TRAIL [31], syncytin-A [32], sclerostin [20], and rIGF-1 $[49,50]$. I provide below a brief discussion of these targets in ascending order of potential.

\section{Tumor necrosis factor-related apoptosis-inducing ligand (TRAIL)}

TRAIL is a cytokine of the TNF superfamily known for its ability to trigger apoptosis in tumor cells while being relatively safe towards normal cells. Binding to its cognate receptors, TRAIL-R1 or TRAIL-R2, induces the formation of a macromolecular complex that recruits caspase- 8 and caspase- 10 to initiate apoptosis [51,52]. Although TRAIL has been shown to trigger osteoclast apoptosis, it is unlikely that this cytokine could be ever be used to target only osteoclasts and titer its use in such a way that the subject does not develop osteopetrosis.

\section{Syncytin-A}

Syncytin- $A$ is an intact retroviral envelope protein, the product of a retrovirus gene that was inserted into the human genome somewhere between 10 and 80 million years ago [53]. Syncytin-A is called a syncytin because of its membrane fusogenic capacity. In bone, syncytin A fuses the membranes of mono-nucleated pre-osteoclasts producing mature multinucleated osteoclasts. However, pre-osteoclast fusion is also mediated by CD47, and syncytin-A plays a critical role in the fusion of the placental 
syncytiotrophoblast [53-55], making this protein an unlikely candidate to target with monoclonal antibody. More suited for this purpose and currently under widespread use are the bisphosphonates, whose effects include osteoclast apoptosis.

Sclerostin

Sclerostin, a 22-kD glycoprotein secreted primarily by osteocytes, is a soluble inhibitor of canonical $W n t / \beta$-catenin signaling pathways that direct mesenchymal stem cells towards the osteoblastic lineage. Sclerostin also enhances bone resorption by decreasing the production of osteoprotegerin, the soluble decoy receptor that inhibits osteoclastogenesis by binding RANKL. Thus, when present in high concentrations sclerostin causes decreased bone formation and increased bone resorption [57].

Scientists have recently developed a humanized monoclonal antibody directed against sclerostin (romosozumab) which was approved by the FDA for treatment of osteoporosis in 2019. Clinical trials have shown that monthly subcutaneous injections of romosozumab is effective in increasing bone formation and density and in decreasing bone resorption, results in keeping with the known effects of sclerostin on bone homeostasis [58]. However, there is some concern about potential cardiotoxicity of romosozumab, prompting the need for further clinical observations [59].

\section{IGF-1}

The potential role of rIGF-1 in maintaining skeletal mass in space was recognized by Tanaka and associates who found that the complex IGF-1 and its specific binding protein, IGFBP-3 stimulated bone turnover in an animal model flown in space [49]. Bateman and associates found that administration of IGF-1 to rodents flown for 10 days aboard the Space Shuttle increased their humerus periosteal bone formation by $37.6 \%[48]$

In light of the central role that IGF-1 plays in all phases of bone formation and preservation, it is perhaps surprising that there are no published proof of principle trials assessing the efficacy of rIGF-1 in preventing bone loss in astronauts, particularly in light of the fact that rIGF-1 is available in an easy to use nasal spray [59]. IGF-1 administration would have the added advantage of bolstering muscle growth and exerting a positive effect on immunoregulation.

It is recognized that IGF1/IGF1Rt studies done under simulated conditions of microgravity do not necessary equate with those done in the zero gravity of space. Simulated microgravity studies achieve at best a thousand-fold reduction in gravitational forces $\left(\geq 1 \times 10^{-3} \mathrm{~g}\right)$ whereas in space the force of gravity is a million-fold less than that experienced on Earth $\left(1 \times 10^{-6} \mathrm{~g}\right)$. As noted in this review, osteoblasts grown in space have extended cell shapes, fragmented and condensed nuclei, shortened microtubules, defective actin microfilaments, smaller and fewer focal adhesions, and even a complete implosion of the cytoskeleton. In contrast, osteoblasts grown in simulated microgravity are characterized more by disruption in functional than in structural aberrations. Thus, although rIGF-1 proved useful in reversing the recalcitrance of blastocytes to mechanostressors in simulated microgravity, the same may not be true in space.

The United States, Russia, and China are planning to establish colonies on our moon sometime in the 2030s. The force of gravity on the Moon is $1.6 \mathrm{~m} / \mathrm{s}^{2}$ which is $16.7 \%$ of Earth's gravity or $\sim 1 \times 10^{-2} \mathrm{~g}$, well within the range of simulated microgravity studies already performed. It would seem that rIFG-1 may 
hold greater promise as a therapy to abrogate hypogravity-related changes in bone homeostasis in astronauts destined to colonize the moon than those aboard a spaceship.

\section{Summary}

1. By tradition bone formation is divided into 4 phases: 1 . Osteoclast differentiation; 2 . Osteoclastmediated bone resorption; 3. Osteoblast-mediated bone formation/mineralization; and 4. Osteoblast to osteocyte transformation and establishment of bone homeostasis.

2. Zero and modeled microgravity adversely affect all 4 phases of bone formation, increasing the maturation and the resorptive activities of osteoclasts, decreasing the differentiation, maturation, and bone forming abilities of osteoblasts, and causing apoptotic-mediated death of osteocytes with consequent dysregulation of bone homeostasis.

3. Microgravity-associated changes in osteoblast microstructure include extended cell shapes, fragmented and condensed nuclei, shortened microtubules, smaller and fewer focal adhesions, and even complete collapse of the cytoskeleton due to microfilament and/or microtubular failure. Osteocyte death is reflected by increases in lacunar vacancies and volumes and in the production of sclerostin, a mechanosensitive protein that inhibits bone formation and enhances bone resorption.

4. IGF-1 and its receptor IGF1R play critical roles in all phases of bone formation, promoting both radial and linear bone growth. Osteocyte and osteoblast responses to mechanostressors is IGF-1/IGF1R dependent and deletion of IGF-1 or IGF1R genes abates their responses to mechanical unloading and reloading. Treatment with recombinant IGF-1 (rIGF1) reverses these defects when IGF1R is intact, raising the prospect that rIGF-1 may prove useful in preventing bone loss associated with space travel or, more likely, in the microgravity of the Moon. Another potential candidate to prevent bone loss in space or on the Moon is romosozumab, a humanized antibody against sclerostin which has been approved by the FDA for treatment of osteoporosis.

\section{Acknowledgements}

The author is grateful for the support of the Division of Academic Affairs in the preparation of this article.

Conflicts of Interest: The author declares no conflict of interest. 


\section{References}

1. Yakar, S.; Werner, H.; Rosen, C.J. Insulin-like growth factors: actions on the skeleton. J. Mol. Endo. 2018, 61, T115-T137.

2. Wittkowske, C.; Reilly, G.C.; Lacroix, D.; Perrault, C.M. In vitro bone cell models: impact of fluid shear stress on bone formation. Front. Bioeng. Biotechnol. 2016, 4, 87. doi: 10.3389/fbioe.2016.00087.

3. Issack, P.S.; Helfet, D.L.; Lane, J.M. Role of Wnt signaling in bone remodeling and repair. HSSJ. 2008, 4, 66-70.

4. Sakai, A. Spaceflight/bedrest immobilization and bone. Osteocyte as a sensor of mechanical stress and Wnt signal. Clin. Calcium. 2012, 22(12), 1829-1835.

5. Aventaggiato, M.; Barreca, F.; Vernucci, E.; Bizzarri, M.; Ferretti, E.; Russo, M.A.; et. al. Putative receptors for gravity sensing in mammalian cells: the effects of microgravity. Appl. Sci. 2020, 10, 2028: doi:10.3390/app10062028.

6. Hillsley, M.V.; Frangos, J.A. Bone tissue engineering: role of interstitial fluid flow. Biotechnol. Bioeng. 1994, 43(7), 573-581.

7. Liu, C.; Zhao, Y.; Cheung, W-Y.; Gandhi, R.; Wang, L.; Lidan, Y. Effects of Cyclic hydraulic pressure on osteocytes. Bone. 2010, 46(S), 1449-1456.

8. Einhorn, T.A. The role of cyclooxygenase-2 in bone repair. Arthritis Res. Ther. 2003, 5, 5-7.

9. Young, S.R.L.; Hum, J.M.; Rodenberg, E.; Turner, C.H.; Pavalko, F.M. Non-overlapping functions for Pyk2 and FAK in osteoblasts during fluid stress-induced mechanotransduction. PLoS ONE. 2011, 6(1), e16026. doi: 10.1371/journal.pone.0016026.

10. Ban, Y.; Wu, Y.; Yu, T.; Geng, N.; Wang, Y.; Liu, X.; et. al. Response of osteoblasts to low fluid shear stress is time dependent. Tissue Cell. 2011, 43 (5), 311-317.

11. Robling, A.G.; Niziolek, P.J.; Baldridge, L.A.; Condon, K.W.; Allen, M.R.; Alam, I.; et. al. Mechanical stimulation of bone in vivo reduces osteocyte expression of Sost/sclerostin. J. Biochem. 2008; 283(9), 5866-5875.

12. Gerbaix, M.; Gnyubkin, V.; Farlay, D.; Olivier, C.; Ammann, P.; Courbon, G.; et. al. One-month spaceflight compromises the bone microstructure, tissue-level mechanical properties, osteocyte survival and lacunae volume in mature mice skeletons. Sci. Rep. 2017, 7(1), 2659. doi: 10.1038/s41598-01703014-2.

13. Rodionova, N.V.; Organov, V.S.; Zolotova, N.V. Ultrastructure changes in osteocytes in microgravity conditions. Adv. Space Res. 2002, 30(4), 765-770.

14. Carmeliet, G.; Bouillon, R. The effect of microgravity on morphology and gene expression of osteoblasts in vitro. FASEB J. 1999, 13, S129-S134.

15. Nabavi, N.; Khandani, A.; Camirand, A.; Harrison, R.E. Effects of microgravity on osteoclast bone resorption and on osteoblast cytoskeletal organization and adhesion. Bone. 2011, 49, 965-974. 
16. Hughs-Fulford, M. Function of the cytoskeleton in gravisensing during spaceflight. Adv. Space Res. 2003, 32, 1583-1593.

17. Hughs-Fulford, M.; Tjandrawinata, R.; Fitzgerald, J.; Gasuad, K.; Gilbertson, V. Effects of microgravity on osteoblast growth. Gravit. Space Biol. Bull. 1998, 11(2), 51-60.

18. Guignandon, A.; Lafage-Proust, M.H.; Usson, Y.; Laroche, N.; Caillot-Augusseau, A.; Alexandre, C.; et. al. Cell cycling determines integrin-mediated adhesion in osteoblastic ROS $17 / 2.8$ cells exposed to spacerelated conditions. FASEB J. 2001, 15(11), 2036-2038.

19. Tamma, R.; Colaianni, G.; Camerino, C.; Di Benedetto, A.; Greco, G.; Strippoli, M.; et. al. Microgravity during spaceflight directly affects in vitro osteoclastogenesis and bone resorption. FASEB J. 2009, 23(8), 2549-2554.

20. Lin, C.; Jiang, X.; Dai, Z.; Guo, X.; Weng, T.; Wang, J.; et. al. Sclerostin mediates bone resorption response to mechanical unloading through antagonizing Wnt/beta-catenin signaling. J. Bone Miner. Res. 2009, 24(10), 1651-1661.

21. Saxena, R.; Pan, G.; McDonald, J.M. Osteoblast and osteoclast differentiation in modeled microgravity. Ann. N. Y. Acad. Sci. 2007, 1116, 494-498.

22. Ontiveros, C., McCabe, L.R. Simulated microgravity suppresses osteoblast phenotype, Runx2 levels and AP-1 transactivation. J. Cell. Biochem. 2003, 88(3), 427-437.

23. Patel, M.J.; Liu, W.; Sykes, M.C.; Ward, N.E.; Risin, S.A., Risin, D.; et. al. Identification of mechanosensitive genes in osteoblasts by comparative microarray studies using the rotating wall vessel and the random positioning machine. J. Cell. Biochem. 2007, 101(3), 587-599.

24. Shi, W.; Xie, Y.; He, J.; Zhou, J.; Gao, Y.; Wei, W.; et. al. Microgravity induces inhibition of osteoblastic differentiation and mineralization through abrogating primary cilia. Sci. Rep. 2017, 7(1), 1866, doi: 10.1038/s41598-017-02049-9.

25. Sun, Z.; Li, Y.; Zhou, H.; Cai, M.; Liu, J.; Gao, S.; et. al. Simulated microgravity reduces intracellularfree calcium concentration by inhibiting calcium channels in primary mouse osteoblasts. J. Cell. Biochem. 2019, 120(3), 4009-4020.

26. Dai, Z.Q.; Wang, R.; Ling, S.K.; Wan, Y.M.; Li, Y.H. Simulated microgravity inhibits the proliferation and osteogenesis of rat bone marrow mesenchymal stem cells. Cell. Prolif. 2007, 40(5), 671-684.

27. Li, L.; Zhang, C.; Chen, J.L.; Hong, F.F.; Chen, P.; Wang, J.F. Effects of simulated microgravity on the expression profiles of RNA during osteogenic differentiation of human bone marrow mesenchymal stem cells. Cell. Prolif. 2019, 52(2): e12539. doi: 10.1111/cpr. 12539.

28. Chen, Z., Luo, Q., Lin, C., Song, G. Simulated microgravity inhibits osteogenic differentiation of mesenchymal stem cells through down regulating the transcriptional co-activator TAZ. Biochem. Biophys. Res. Commun. 2015, 468(1-2), 21-26.

29. Michaletti, A.; Gioia, M.; Tarantino, U.; Zolla, L. Effects of microgravity on osteoblast mitochondria: a proteomic and metabolomics profile. Scientific reports. 2017. doi: 10.1038/s41598-017-15612-1. 
30. Sambandam, Y.; Townsend, M.T.; Pierce, J.J.; Lipman, C.M.; Haque, A.; Bateman, T.A.; et. al. Microgravity control of autophagy modulates osteoclastogenesis. Bone. 2014, 61, 125-131.

31. Sambandam, Y.; Baird, K.L.; Stroebel, M.; Kowai, E.; Balasubramanian, S.; Reddy, S.V. Microgravity induction of TRAIL expression in preosteoclast cells enhances osteoclast differentiation. Sci. Rep. 2016, 6,25143. doi: 10.1038/srep25143.

32. Ethiraj, P.; Link, J.R.; Sinkway, J.M.; Brown, G.D.; Parler, W.A.; Reddy, S.V. Microgravity modulation of syncytin-A expression enhance osteoclast formation. J. Cell. Biochem. 2018, 119(7), 5696-5703.

33. Rucci, N.; Rufo, A.; Alamanou, M.; Teti, A. Modeled microgravity stimulates osteoclastogenesis and bone resorption by increasing osteoblast RANKL/OPG ratio. J. Cell. Biochem. 2007, 100(2), 464-473.

34. Saxena, R.; Pan, G.; Dohm, E.D.; McDonald, J.M. Modeled microgravity and hindlimb unloading sensitize osteoclast precursors to RANKL-mediated osteoclastogenesis. J. Bone Miner. Metab. 2011, 29(1), 111-122.

35. Li, J.; Choi, E.; Yu, H.; Bai, X.C. Structural basis of the activation of type 1 insulin-like growth factor receptor. Nat. Commun. 2019, 10(1), 4567. doi: 10.1038/s41467-019-12564-0.

36. Zhang, X.; Yu, D.; Sun, J.; Wang, Y.; Ouyang, O.; Wang, T. Visualization of ligand-bound ectodomain assembly in the full-length human IGF-1 receptor by cryo-EM single-particle analysis. Structure. 2020, 28, 1-7.

37. Yakar, S.; Rosen, C.J.; Beamer, W.G.; Ackert-Bicknell, C.L.; Wu, Y.; Liu, J-L.; et. al. Circulating levels of IGF-1 directly regulate bone growth and density. J. Clin. Invest. 2002, 110, 771-781.

38. Janssen, J.A. New insights from IGF-1R stimulating activity analysis: pathological considerations. Cells. 2020, 9, 862, doi:10.3390/cells/9040862.

39. Crane, J.L.; Cao, X. Function of matrix IGF-1 in coupling bone resorption and formation. J. Mol. Med. 2014, 92(2), 107-115.

40. Clarmatori, S.; Kiepe, D.; Haarmann, A.; Huegel, U.; Tonshoff, B. Signaling mechanisms leading to regulation of proliferation and differentiation of the mesenchymal chondrogenic cell line. J. Mol. Endocrinol. 2007, 38,493-508.

41. Xian, L.; Wu, X.; Pang, L.; Lou, M.; Rosen, C.; Qiu, T.; et. al. Matrix IGF-1 regulates bone mass by activation of mTOR in mesenchymal stem cells. Nat. Med. 2012, 18(7), 1095-1101.

42. Kostenuik, P.J.; Harris, J.; Halloran, B.P.; Turner, R.T.; Morey-Holton, E.R.; Bikle, D.D. Skeletal unloading causes resistance of osteoprogenitor cells to parathyroid hormone and to insulin-like growth factor-1. J. Bone Miner. Res. 1999, 14(1), 21-31.

43. Wang, Y.; Nishida, S.; Elalieh, H.Z.; Long, R.K.; Halloran, B.P.; Bikle, D.D. Role of IGF-1 signaling in regulating osteoclastogenesis. J. Bone Min. Res. 2006, 21(9), 1350-1358.

44. Pavalko, F.M.; Gerard, R.L.; Ponik, S.M.; Gallagher, P.J.; Jin, Y.; Norvell, S.M. Fluid shear stress inhibits TNF-alpha-induced apoptosis in osteoblasts: a role for fluid shear stress-induced activation of PI3-kinase and inhibition of caspase-3. J. Cell. Physiol. 2003, 194(2), 194-205. 
45. Triplett, J.W.; O’Riley, R.; Tekulve, K.; Norvell, S.M.; Pavalko, F.M. Mechanical loading by fluid shear stress enhances IGF-1 receptor signaling in osteoblasts in a PKCzeta-dependent manner. Mol. Cell. Biomech. 2007, 4(1), 13-25.

46. Tian, F.; Wang, Y.; Bickle, D.D. IGF-1 signaling mediated cell-specific skeletal mechano-transduction. J. Orthop. Res. 2018, 36(2), 576-583.

47. Kumei, Y.; Nakamura, H.; Morita, S.; Akiyama, H.; Hirano, M.; Ohya, K.; et. al. Space flight and insulinlike growth factor signaling in rat osteoblasts. Ann. N. Y. Acad. Sci. 2002, 973, 75-78.

48. Sakata, T.; Wang, Y.; Halloran, B.P.; Elalieh, H.Z.; Cao, J.; Bikle, D.D. Skeletal unloading induces resistance to insulin-like growth factor-1 (IGF-1) by inhibiting activation of the IGF-1 signaling pathways. J. Bone Miner. Res. 2004, 19(3), 436-446.

49. Tanaka, H.; Moriwake, T.; Matsuoka, Y.; Nakamura, T.; Seino, Y. Potential role of rhIGF-1/IGFBP-3 in maintaining skeletal mass in space. Bone. 1998, 22 (5 Suppl), 145S-147S.

50. Bateman, T.A.; Zimmerman, R.J.; Ayers, R.A.; Ferguson, V.L.; Chapes, S.K.; Simske, S.J. Histomorphometric, physical, and mechanical effects of spaceflight and insulin-like growth factor on rat long bones. Bone. 1998, 23(6), 527-35.

51. Micheau, O. Regulation of TNF-related apoptosis-inducing ligand signaling by glycosylation. Int. J. Mol. Sci. 2018, 19(3), pii:E715. doi: 10:3390/ijms19030715.

52. Walczak, H. Biochemical analysis of the native TRAIL death-inducing signaling complex. Methods Mol. Biol. 2008, 414, 221-239.

53. Dupressoir, A., Lavialle, G., Heidmann, T. From ancestral infectious retroviruses to bone fide cellular genes: role of the captured syncytins in placentation. Placenta. 2012, 33(9), 663-671.

54. Peng, X., Pan, J., Gong, R., Liu, Y., Kang, S., Feng, H., et. al. Functional characterization of syncytin-A, a newly murine endogenous virus protein. Implication for its fusion mechanism. J. Biol. Chem. 2007, 282(1), 381-389.

55. Moller, A.M., Delaissé, J.M., Soe, K. Osteoclast fusion: time-lapse reveals involvement of CD47 and syncytin-1 at different stages of nuclearity. J. Cell. Physiol. 2017, 232(6), 1396-1403.

56. De Maré, A., D'Haese, P.C., Verhulst, A. The role of sclerostin in bone and ectopic calcification. Int. J. Mol. Sci. 2020, 21, 3199, doi: 10.3390/ijms21093199.

57. Bandeira, L., Lewiecki, E.M., Bilezikian, J.P. Romosozumab for the treatment of osteoporosis. Expert Opin. Biol. Ther. 2017, 17(2), 255-263.

58. Shakeri, A., Adanty, C. Romosozumab (sclerostin monoclonal antibody) for the treatment of osteoporosis in postmenopausal women: a review. J. Popul. Ther. Clin. Pharmacol. 2020, 27(1): e25-e31. doi: 10.15586/jptcp.v2711.655. 
59. Liu, X.F., Fawcett, J.R., Thome, R.G., DeFor, T.A., Frey, W.H. 2nd. Intranasal administration of insulinlike growth factor-1 bypasses the blood-brain barrier and protects against focal cerebral ischemic damage. J. Neurol. Sci. 2001, 187 (1-2), 91-97. 